\title{
Comparison of tenofovir plus lamivudine versus tenofovir monotherapy in patients with lamivudine- resistant chronic hepatitis B
}

\author{
Chan Ho Park, Seok Won Jung, Jung Woo Shin, Mi Ae Bae, Yoon Im Lee, Yong Tae Park, Hwa Sik Chung, \\ and Neung Hwa Park \\ Department of Internal Medicine, University of Ulsan College of Medicine, Ulsan University Hospital, Ulsan, Korea
}

Background/Aims: Tenofovir disoproxil fumarate (TDF) exhibits similar antiviral efficacy against treatment-naïve and lamivudine (LAM)-resistant chronic hepatitis B (CHB). However, there are few clinical reports on the antiviral effects of TDF-LAM combination therapy compared to TDF monotherapy in patients with LAM-resistant CHB.

Methods: We investigated the antiviral efficacy of TDF monotherapy vs. TDF-LAM combination therapy in 103 patients with LAM-resistant CHB.

Results: The study subjects were treated with TDF alone $(n=40)$ or TDF-LAM combination therapy $(n=63)$ for $\geq 6$ months. The patients had previously been treated with TDF-based rescue therapy for a median of 30.0 months (range, 8-36 months). A virologic response (VR) was achieved in 99 patients (96.1\%): 95.0\% (38/40) of patients in the TDF monotherapy group and $96.8 \%$ (61/63) of patients in the TDF-LAM combination therapy group. The VR rates were not significantly different between the TDF monotherapy and TDF-LAM combination therapy groups (88.9 vs. $87.3 \%$ at month 12 , and 94.4 vs. $93.7 \%$ at month 24 , log-rank $P=0.652$ ). Univariate and multivariate analyses revealed that none of the pretreatment factors were significantly associated with VR.

Conclusions: TDF monotherapy was as effective as TDF-LAM combination therapy for maintaining viral suppression in the vast majority of patients with LAM-resistant CHB, which suggests that TDF add-on therapy with LAM is unnecessary. (Clin Mol Hepatol 2016;22:152-159)

Keywords: Tenofovir; Lamivudine; Resistance; Chronic hepatitis B

\section{INTRODUCTION}

Hepatitis B virus (HBV) infection is a serious public health problem worldwide, with an estimated 2 billion infections, and a major cause of chronic hepatitis, cirrhosis, and hepatocellular carcinoma (HCC). ${ }^{1}$ Higher levels of HBV DNA are associated with an increased risk for hepatocellular carcinoma (HCC) and cirrhosis., ${ }^{2,3}$ Thus, lowering of HBV DNA levels from the highest levels has been linked with a reduction in risk of both cirrhosis and $\mathrm{HCC}^{4-7}$ Therefore, the goal of therapy in patients with chronic hepatitis B $(\mathrm{CHB})$ is rapid and sustained viral suppression.

Nucleos(t)ide analogues (NAs) as an important class of antiviral

\section{Abbreviations:}

ADV, adefovir; AE, adverse event; CHB, chronic hepatitis B; HBsAg, hepatitis B surface antigen; HBV, hepatitis B virus; HCC, hepatocellular carcinoma; LAM, lamivudine; PCR-RFLP, polymerase chain reactionrestriction fragment length polymorphism; PVR, partial virologic response; RFMP, restriction fragment mass polymorphism; TDF, tenofovir disoproxil fumarate; VBT, virologic breakthrough; VR, virologic response
}

\section{Corresponding author : Neung Hwa Park}

Department of Internal Medicine, Ulsan University Hospital, 877 Bangeojinsunhwando-ro, Dong-gu, Ulsan 44033, Korea

Tel: +82-52-250-8845, Fax: +82-52-250-7048

E-mail:nhpark@uuh.ulsan.kr 
drugs have changed the treatment paradigm and prognosis of CHB. Lamivudine (LAM), the first oral antiviral agent approved to treat $H B V$, is safe and well tolerated, even in patients with decompensated liver cirrhosis. ${ }^{8}$ However, long-term use of NAs inevitably leads to the development of resistant HBV mutants and viral breakthrough. Resistance to LAM emerges in approximately $20 \%$ of patients after 1 year and in $70 \%$ of patients after 5 years of treatment. ${ }^{9}$ Continued treatment with LAM monotherapy in patients with LAM-resistant CHB is not recommended due to liver disease progression, including worsening of fibrosis and cirrhosis, and selection of secondary mutations. ${ }^{10}$ In addition, an increasing number of patients experience multiple NAs treatment failures, especially when they are sequentially treated with NAs that have low genetic barrier such as LAM and ADV. ${ }^{11-13}$ Therefore, for CHB patients with LAM resistance, current international guidelines recommend switching to tenofovir disoproxil fumarate (TDF), or adding on TDF. ${ }^{14,15}$

TDF achieved high rates of virologic suppression, with no development of genotypic resistance reported so far. ${ }^{16}$ TDF is recommended as first-line therapy in patients with $\mathrm{CHB} .{ }^{14,15}$ It is also recommended for patients who have developed resistance to LAM. TDF is active in vitro against both wild-type and LAM-resistant $H B V .^{17}$ Several studies have shown that the efficacy of TDF monotherapy for treating of patients with LAM-resistant CHB is currently limited to small case series or retrospective clinic-based studies. ${ }^{18-21}$ In addition, recent data show that TDF rescue therapy with LAM or emtricitabine retains significant activity for patients with LAM-resistant $\mathrm{HBV}^{22-24}$ Fung et al. studied the efficacy of TDF/emtricitabine combination therapy compared to that of TDF monotherapy in patients with LAM-resistant $\mathrm{CHB} .{ }^{25}$ That study showed that TDF monotherapy was as effective as the combination of TDF/emtricitabine in patients with LAM-resistant CHB. However, clinical experience with the antiviral effect of TDF-LAM combination therapy compared to TDF monotherapy in patients with LAM-resistant CHB is limited. In this study, we evaluated the efficacy of TDF-LAM combination therapy compared to that of TDF monotherapy in patients who developed resistance to LAM. We also determined patient-dependent or laboratory variables that predicted VR.

\section{PATIENTS AND METHODS}

\section{Patient population}

We reviewed the electronic medical records of patients with
LAM-resistant CHB and who were treated with TDF-based rescue therapy for at least 6 months. A total of 103 patients were treated with TDF alone or a LAM combination by clinician or patient choice from December 2012 to November 2015. The study subjects were treated with TDF alone $(n=40)$ or a TDF-LAM combination ( $n=63$ ) for $\geq 6$ months. All patients had hepatitis B surface antigen (HBsAg) and HBV DNA in serum for at least 6 months before the start of LAM therapy. Patients with impaired renal function (serum creatinine $>1.5 \mathrm{mg} / \mathrm{dL}$ ), antibodies to hepatitis C virus, antibodies to HIV, or autoimmune hepatitis were excluded. Additional criteria for exclusion were pregnancy, lactation, and alcohol abuse (> $40 \mathrm{~g} /$ day ethanol). Diagnoses of chronic hepatitis and liver cirrhosis were based on liver biopsy features or on clinical, laboratory, and ultrasound data. The diagnosis of CHB was based on histological examination for 16 patients. The remaining 87 patients were clinically diagnosed. Written informed consent was obtained from all patients participating in this study. This research was approved by the Institutional Review Board at the UIsan University Hospital.

\section{Laboratory measurements}

Liver and kidney function tests were performed every 3 months during TDF rescue therapy. HBV DNA levels were quantified using the COBAS TaqMan HBV test (Roche, Branchburg, NJ, USA), which has a lower detection limit of $12 \mathrm{IU} / \mathrm{mL}$ (60 copies/mL). Specific HBV genotypes were identified using polymerase chain reaction-restriction fragment length polymorphism (PCR-RFLP) analysis of the surface gene of HBV genome. The two fragments of HBV genome between nucleotide positions 2823 and 2845 and 61 and 80 were amplified using PCR, and the products were treated with restriction enzymes. Genotypic resistance to LAM was tested by restriction fragment mass polymorphism (RFMP; Genematrix, Seongnam-si, Gyeonggi, Korea assay). The RFMP assay can detect 100 copies of HBV genome per milliliter. Patients underwent surveillance for HCC every 6 months, and serial abdominal ultrasound and serum a-fetoprotein measurements were performed.

\section{Definitions}

Virologic response (VR) was defined as the absence of serum HBV DNA by $P C R$ assay $(<12 \mathrm{IU} / \mathrm{mL})$ on two consecutive measurements during TDF treatment. HBeAg seroconversion was defined as the loss of HBeAg accompanied by detection of anti-HBe 
and the absence of serum HBV DNA during treatment. Partial virologic response (PVR) was defined as a decrease in HBV DNA of $>1 \log _{10} \mathrm{IU} / \mathrm{mL}$ but detectable HBV DNA after 6 months of TDFbased rescue therapy. Virologic breakthrough (VBT) was defined as a $>1 \log _{10} \mathrm{IU} / \mathrm{mL}$ increase in serum HBV DNA from the nadir on two consecutive measurements or on the last available measurement. Safety and tolerability were evaluated by the occurrence of adverse events (AEs), serious AEs, laboratory abnormalities, discontinuation of the study drug due to AEs, or death. Specific markers of renal abnormalities included confirmed (defined as two consecutive visits) increase in serum creatinine of at least $0.5 \mathrm{mg} / \mathrm{dL}$ above the baseline value, serum phosphorus values $<2 \mathrm{mg} / \mathrm{dL}$, and creatinine clearance $<50 \mathrm{~mL} / \mathrm{min}$.

\section{Statistical analysis}

Serum HBV DNA (IU/mL) levels were logarithmically transformed for analysis. Continuous variables were compared using Student's $t$ test, and categorical variables were compared using the $\chi^{2}$ test. The cumulative probability rates of clinical outcomes were calculated using the Kaplan-Meier method. To identify factors predictive of outcome among the baseline variables, the clinical outcome variables were compared using the $\chi^{2}$ test or univariate logistic regression. Multivariate analysis was carried out using a stepwise logistic regression model. All data were analyzed using the SPSS ver. 19.0 for Windows statistical package (SPSS Inc., Chicago, IL, USA). A two-tailed $P$-value $<0.05$ was considered significant.

\section{RESULTS}

\section{Overall clinical outcomes of TDF-based therapy}

The baseline characteristics of the 103 patients with CHB are shown in Table 1. All patients were genotype C2. The patients had been treated with LAM for a median of 33.0 months (range, 7-151 months) prior to TDF-based rescue treatment. All patients had LAM-resistant CHB. The known genotypic LAM mutations, L180M M204V, L180M M204I, L180M M204I/V, M204I, and M204V were detected in 44, 19, 12, 26, and two patients, respectively. Thirty-four (33.0\%) patients had liver cirrhosis. Seventy-eight (75.7\%) patients were HBeAg positive. The median HBV DNA level prior to TDF-based treatment was $3.61 \log _{10} \mathrm{IU} / \mathrm{mL}$ (range, $1.41-8.23 \log _{10} \mathrm{IU} / \mathrm{mL}$ ). Thirty-four (33.0\%) patients had abnormal alanine aminotransferase (ALT) levels. The subjects
Table 1. Baseline characteristics of the studied patients $(n=103)$

\begin{tabular}{lc}
\hline Age (year) & $51(27-90)$ \\
Sex (male/female) & $79 / 24$ \\
Liver cirrhosis, n (\%) & $34(33.0)$ \\
AST (IU/L) & $27.0(15-2,431)$ \\
ALT (IU/L) & $30.0(6-3,248)$ \\
HBV DNA (log 10 IU/mL) & $3.61(1.41-8.23)$ \\
HBeAg positivity, n (\%) & $78(75.7)$ \\
Duration of LAM therapy (months) & $33(7-151)$ \\
Duration of TDF therapy (months) & $30(8-36)$ \\
\hline LAM resistant mutations & \\
\hline L180M M204V, L180M M204I, L180M M204I/V, & 44,19, \\
M204l, M204V & $12,26,2$ \\
\hline
\end{tabular}

Continuous variables are expressed as medians with range.

AST, aspartate aminotransferase; ALT, alanine aminotransferase; HBV, hepatitis $B$ virus; HBeAg, hepatitis $B$ e antigen; LAM, lamivudine; TDF, tenofovir.

were treated with TDF alone $(n=40)$ or the TDF-LAM combination $(n=63)$ for $\geq 6$ months. The patients had been treated with TDFbased rescue therapy for a median of 30.0 months (range, 8-36 months). Mean pre-treatment HBV DNA level in HBeAg-positive CHB patients was $3.80 \pm 1.58 \log _{10} \mathrm{IU} / \mathrm{mL}$, and it was $4.38 \pm 1.51$ $\log _{10} \mathrm{IU} / \mathrm{mL}$ in HBeAg-negative CHB patients. Mean pre-treatment HBV DNA level in HBeAg-negative CHB patients was similar to that in HBeAg-positive CHB patients $(P=0.109)$. Among the 34 patients with elevated ALT levels at baseline, ALT levels were normalized in 31 patients (91.2\%) during TDF-based treatment. VR was achieved in 99 patients (96.1\%) during TDF-based rescue therapy. The cumulative rates of VR at 6,12 , and 24 months were $77.7 \%, 87.8 \%$, and $93.9 \%$, respectively. During TDF-based rescue therapy, 21 (20.4\%) patients had PVR. Among the $78 \mathrm{HBeAg}$ positive patients, seven (9.0\%) patients achieved HBeAg seroconversion during TDF-based treatment. However, no patient lost serum HBsAg during treatment. One patient in the TDF monotherapy group experienced VBT (increase in HBV DNA by 1 $\log _{10} \mathrm{IU} / \mathrm{mL}$ at 18 months of treatment) associated with documented nonadherence to medication (determined by review of medical and pharmacy records). This patient with VBT responded well to TDF monotherapy after continuation of treatment.

No clinically significant AEs were observed during the TDFbased treatment. Mean creatinine level and estimated glomerular filtration rate did not change during the treatment period. Two patients (1.9\%) had an increase in serum creatinine levels, but no patient had an increase in serum creatinine $>0.5 \mathrm{mg} / \mathrm{dL}$. Mild hypophosphatemia (serum phosphorus $<2.7 \mathrm{mg} / \mathrm{dL}$ ) was found in 
only one patient without a change in serum creatinine.

\section{Comparison of clinical outcomes between the TDF- LAM combination and TDF monotherapy groups}

The baseline characteristics of the TDF monotherapy $(n=40)$ and TDF-LAM combination groups $(n=63)$ are shown in Table 2 . There was no significant difference between the two groups except for baseline HBV DNA levels and HBeAg positivity. The patients in the TDF monotherapy group had significantly higher HBV DNA levels than the patients in the TDF-LAM combination therapy group $(P<0.001)$. The rate of HBeAg positivity was significantly higher in the TDF-LAM combination therapy group than in the TDF monotherapy group $(P=0.004)$. The overall clinical outcomes between the TDF monotherapy and TDF-LAM combination groups are summarized in Table 3. VR was observed in 95.0\% (38/40) of patients in the TDF montherapy group and in 96.8\% (61/63) of patients in the TDF-LAM combination group. The VR rates were compared using a log-rank test to define whether there was any difference in the VR rates between the TDF-LAM combination and TDF monotherapy groups. The rates of VR were not significantly different between the TDF monotherapy and TDF-LAM combination groups (88.9 vs. $87.3 \%$ at month 12 , and 94.4 vs. $93.7 \%$ at month 24; Fig. 1; log-rank test, $P=0.652$ ). Additionally, the proportion of patients that achieved a normalized ALT level was also similar between the groups. During TDF rescue therapy, 16.7\% (4 of 24) of patients in the TDF monotherapy group, and 5.6\% (3 of $54)$ of patients in the TDF-LAM combination group achieved HBeAg seroconversion, respectively $(P=0.113)$. VBT occurred in only one patient (none in the TDF-LAM combination group and

Table 2. Baseline characteristics of patients in the TDF monotherapy and TDF-LAM combination therapy groups

\begin{tabular}{|c|c|c|c|}
\hline & TDF monotherapy $(n=40)$ & TDF-LAM combination $(n=63)$ & $P$-value \\
\hline Age (years) & $49.5 \pm 11.8$ & $49.9 \pm 9.1$ & 0.858 \\
\hline Sex (male/female) & $28 / 12$ & $51 / 12$ & 0.236 \\
\hline Liver cirrhosis, n (\%) & $15(37.5)$ & $19(30.2)$ & 0.520 \\
\hline AST (IU/L) & $101.8 \pm 378.9$ & $36.1 \pm 51.2$ & 0.176 \\
\hline ALT (IU/L) & $140.2 \pm 508.2$ & $49.2 \pm 90.7$ & 0.167 \\
\hline HBV DNA $\left(\log _{10} I \mathrm{IU} / \mathrm{mL}\right)$ & $4.73 \pm 1.75$ & $3.44 \pm 1.23$ & $<0.001$ \\
\hline HBeAg positivity, n (\%) & $24(60.0)$ & $54(85.7)$ & 0.004 \\
\hline Duration of LAM therapy (months) & $42.7 \pm 38.9$ & $30.2 \pm 22.5$ & 0.187 \\
\hline Duration of TDF therapy (months) & $27.5 \pm 5.6$ & $28.6 \pm 4.7$ & 0.250 \\
\hline LAM resistant mutations & & & 0.331 \\
\hline L180M M204V & 16 & 28 & \\
\hline L180M M204I & 9 & 10 & \\
\hline L180M M204I/V & 5 & 7 & \\
\hline M204I & 8 & 18 & \\
\hline M204V & 2 & 0 & \\
\hline
\end{tabular}

Continuous variables are expressed as means \pm standard deviations.

AST, aspartate aminotransferase; ALT, alanine aminotransferase; HBV, hepatitis B virus; HBeAg, hepatitis B e antigen; LAM, lamivudine; TDF, tenofovir.

Table 3. Comparison of overall clinical outcomes between the TDF monotherapy and TDF-LAM combination therapy groups

\begin{tabular}{lccc}
\hline & TDF monotherapy $(\mathbf{n}=\mathbf{4 0})$ & TDF-LAM combination $(\mathbf{n}=\mathbf{6 3})$ & $P$-value \\
\hline ALT normalization, $n(\%)$ & $18 / 20(90.0)$ & $13 / 14(92.9)$ & 0.773 \\
VR, $n(\%)$ & $38(95.0)$ & $61(96.8)$ & 0.641 \\
\hline HBeAg seroconversion, rate (\%) & $4 / 23(16.7)$ & $3 / 54(5.6)$ & 0.113 \\
PVR, $n(\%)$ & $9(22.5)$ & $12(19.0)$ & 0.803 \\
VBT, $n(\%)$ & $1(2.5)$ & $0(0.0)$ & 0.822 \\
\hline
\end{tabular}

ALT, alanine aminotransferase; VR, virologic response; HBeAg, hepatitis B e antigen; PVR, partial virologic response;VBT, virological breakthrough; TDF, tenofovir; LAM, lamivudine. 
one in the TDF monotherapy group) during the treatment period.

To determine whether there was any difference in the VR rates according to clinical and virological factors at the beginning of TDF rescue therapy, such as baseline HBV DNA levels, genotypic resistance profile, or TDF monotherapy vs. TDF-LAM combination therapy, the VR rates were compared according to these variables using a logistic regression analysis (Table 4). Among the pretreatment factors, any variable was not significantly associated with VR in the univariate and multivariate analyses. That is, the treatment efficacy of TDF-based rescue therapy was not different according to baseline HBV DNA levels and LAM mutation profile.

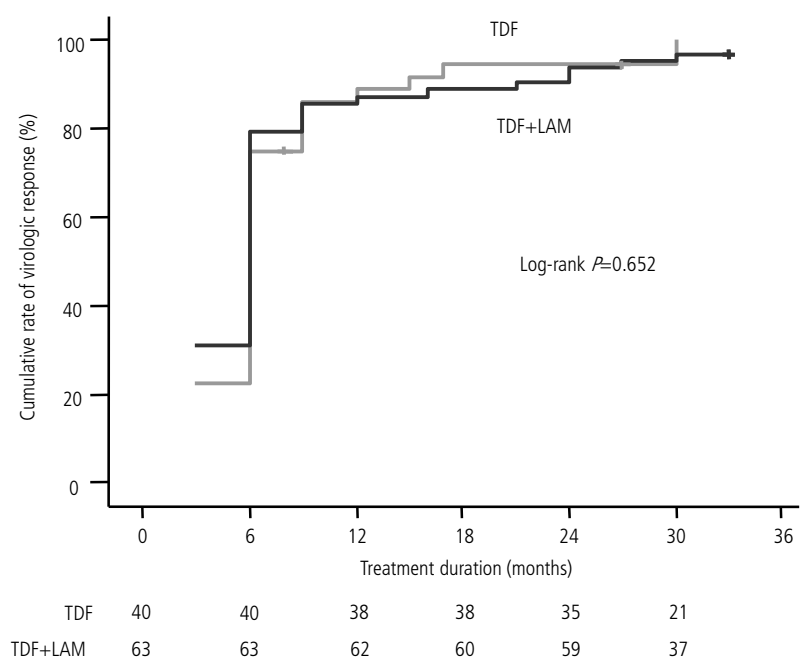

Figure 1. The cumulative VR rate did not differ significantly between the TDF monotherapy and TDF-LAM combination therapy groups (88.9 vs. $87.3 \%$ at month 12 , and 94.4 vs. $93.7 \%$ at month 24 ; log-rank test, $P=0.652)$. VR, virologic response; TDF, tenofovir; LAM, lamivudine.

\section{DISCUSSION}

Very limited data are available on the comparative of efficacy of TDF monotherapy and TDF-LAM combination therapy in patients with LAM-resistant CHB. In our study, TDF monotherapy was as effective as TDF-LAM combination therapy for maintaining viral suppression in patients with LAM-resistant CHB. We also found that long-term TDF-based rescue therapy could lead to viral suppression in the vast majority of LAM-resistant CHB patients, regardless of pretreatment factors at the beginning of TDF rescue therapy.

TDF is active against wild-type and LAM-resistant HBV in vitro. ${ }^{17}$ Therefore, the current international guidelines for patients with LAM-resistant CHB recommend switching to TDF, or adding on TDF. $^{14,15}$ Although data are from small sample sizes or retrospective clinic-based studies, TDF alone is safe and effective for treating patients with LAM-resistant $\mathrm{CHB} .{ }^{20,21}$ A study by van Bömmel et al included 70 patients with LAM-resistant CHB. Overall, 79\% of the patients achieved an HBV DNA level $<80 \mathrm{IU} / \mathrm{mL}$ after a mean TDF monotherapy duration of 23 months. ${ }^{20}$ Those authors reported substantial efficacy regardless of LAM resistance. In addition, other studies have shown that TDF-based combination therapy with LAM or emtricitabine retains significant activity for patients with LAM-resistant HBV. ${ }^{22-24}$ Fung et al randomized 280 patients with LAM-resistant CHB to TDF/emtricitabine combination and TDF monotherapy groups. At treatment week 96, 89.4\% of patients in the TDF group $(n=139)$ and $86.3 \%$ in the TDF/emtricitabine group $(n=141)$ had HBV DNA levels $<69 \mathrm{IU} / \mathrm{mL}$ $(P=0.43) .{ }^{25}$ The authors reported similar efficacies in both the

Table 4. Analysis of the predictive factors for a VR

\begin{tabular}{|c|c|c|c|c|c|c|}
\hline & \multicolumn{3}{|c|}{ Univariate analysis } & \multicolumn{3}{|c|}{ Multivariate analysis } \\
\hline & OR & $95 \% \mathrm{Cl}$ & $P$-value & OR & $95 \% \mathrm{Cl}$ & $P$-value \\
\hline Age & 0.966 & $0.871-1.071$ & 0.507 & 0.940 & $0.837-1.055$ & 0.293 \\
\hline Gender & 0.908 & $0.090-9.153$ & 0.935 & 0.471 & $0.064-0.473$ & 0.461 \\
\hline Diagnosis (CH vs. LC) & 0.492 & $0.053-4.584$ & 0.534 & 0.460 & $0.029-7.389$ & 0.583 \\
\hline Duration of TDF therapy & 0.923 & $0.803-1.060$ & 0.257 & 0.950 & $0.820-1.102$ & 0.499 \\
\hline AST & 0.999 & $0.992-1.007$ & 0.883 & 1.025 & $0.943-1.115$ & 0.560 \\
\hline ALT & 0.999 & $0.992-1.007$ & 0.862 & 0.980 & $0.922-1.043$ & 0.527 \\
\hline HBeAg positivity & 0.960 & $0.095-9.665$ & 0.972 & 0.555 & $0.040-7.633$ & 0.660 \\
\hline Pretreatment HBV DNA level & 1.174 & $0.691-1.993$ & 0.553 & 1.265 & $0.610-2.622$ & 0.527 \\
\hline LAM mutation profile & 1.586 & $0.252-9.998$ & 0.623 & 1.629 & $0.238-11.178$ & 0.619 \\
\hline Rescue therapy regimen (TDF vs. TDF-LAM) & 0.950 & $0.152-5.950$ & 0.956 & 0.688 & $0.075-6.337$ & 0.741 \\
\hline
\end{tabular}

CH, chronic hepatitis; LC, liver cirrhosis; TDF, tenofovir; AST, aspartate aminotransferase; ALT, alanine aminotransferase; HBeAg, hepatitis B e antigen; HBV, hepatitis B virus; LAM, lamivudine; $\mathrm{OR}$, odds ratio; $\mathrm{Cl}$, confidence interval. 
combination and monotherapy groups, and the TDF response was not influenced by the presence of any resistance mutation. A prospective study by Patterson et al included 20 patients with LAMresistant HBV infection. ${ }^{24}$ At the end of the 96 week follow-up, $64 \%$ of the patients achieved an undetectable viral load ( $<15$ IU/ $\mathrm{mL}$ ). In our study, VR occurred in $96.1 \%$ of patients after a median TDF rescue therapy duration of 30 months. The cumulative rates of $V R$ at 6,12 , and 24 months were $77.7 \%, 87.8 \%$, and $93.9 \%$, respectively. Our results compare favorably with previous studies conducted in patients with LAM-resistant CHB. However, clinical experience with the antiviral effect of TDF-LAM combination therapy compared to TDF monotherapy in patients with LAM-resistant CHB is limited. Therefore, we evaluated the efficacy of TDF-LAM combination therapy compared to that of TDF monotherapy in patients with LAM resistance. VR was observed in $95.0 \%$ (38/40) of patients in the TDF montherapy group and in $96.8 \%(61 / 63)$ of patients in the TDF-LAM combination group. That is, no difference was observed between the TDF and TDF-LAM groups. In addition, treatment efficacy of TDF-LAM combinatio or TDF monotherapy was not different according to the LAM mutation profile. We confirmed that add-on combination therapy does not provide any additive suppressive effect on LAM-resistant CHB, which agrees with studies reported previously. A simpler regimen, such as TDF monotherapy, may offer some advantage for reducing non-adherence compared to combination therapy with two medications, such as LAM and TDF. In addition to a lower rate of non-adherence, monotherapy also has significant advantages of lower cost and a lower rate of long-term side effects compared to those of combination therapy. Given the long-term nature of antiHBV therapy, cost reduction is a major concern. However, drugresistance has been a serious clinical challenge in CHB treatment, particularly for patients with NA-resistant CHB. Sequential NA monotherapy promotes selection of multidrug-resistant mutations. Insufficient antiviral efficacy caused by drug resistance results in attenuated viral suppression that may lead to significant clinical deterioration. ${ }^{14,15} \mathrm{~A}$ randomized controlled trial is needed to evaluate the efficacy and resistance of TDF-based rescue therapy in patients with LAM-resistant HBV.

Many pretreatment variables have been investigated to identify predictive factors for selecting patients most likely to respond to therapy. ${ }^{26-29}$ From the outset of HBV antiviral therapy, it has been conventionally accepted that patients with lower baseline viral levels are more responsive than patients with higher viral levels. In TDF-naive patients, Gordon et al. have explored the prognostic significance of baseline serum HBV DNA levels; CHB patients with high viral load can achieve VR at similar rates as patients with lower viral loads, but VR tends to take longer in high viral load patients. ${ }^{30}$ However, few data are available regarding the clinical variables predicting VR during TDF-based rescue therapy in patients with LAM-resistant HBV. Thus, we determined the impact of clinical factors on VR. Among the pretreatment factors at the beginning of TDF rescue therapy, any variable was not significantly associated with VR in the univariate and multivariate analyses. The reason for these results seem the vast majority of patients in our study, whether high or low HBV DNA levels, were achieved VR with extended TDF therapy. Our results are in accordance with previous reported study of TDF therapy in NA naïve CHB patients. ${ }^{30}$ Accordingly, HBV DNA levels should not be a predictor of VR in TDF therapy, regardless of NA-resistant HBV.

The safety profile of TDF in this study was similar to that reported in clinical trials and other field studies performed in a wider patient population. ${ }^{16,31}$ There were no unexpected AEs and the treatment was well-tolerated. Importantly, none of the patients showed a confirmed elevation in serum creatinine level of $>0.5$ $\mathrm{mg} / \mathrm{dL}$ from baseline, and none of the patients developed severe renal complications. One patient in TDF monotherapy group experienced VBT, and this was due to the lack of adherence to medication. Medication adherence has been reported to be an important factor for VBT. ${ }^{32-34}$

This study had several important limitations. The study design was retrospective, in spite of a relatively large sample size and long-term follow-up period. Another limitation of our study was that TDF-resistant mutational analysis in patients with continued viremia or who experienced VBT was not measured. Moreover, we did not assay HBsAg quantitation. Further larger cohort studies with longer follow-up duration are warranted to verify the efficacy and safety of a TDF-based recue regimen.

In conclusion, TDF based rescue therapy was well tolerated without significant adverse events such as renal toxicity in patients with LAM-resistant CHB. TDF monotherapy was as effective as TDFLAM combination therapy for maintaining viral suppression in the vast majority of patients with LAM-resistant CHB. Therefore, addon therapy with LAM appeared to be unnecessary, because it did not provide further benefit over TDF alone regarding VR.

\section{Acknowledgments}

This study was supported by Priority Research Center Program through the National Research Foundation of Korea (NRF) funded by the Ministry of Education, Science, and Technology (20090094050) 


\section{Conflicts of Interest}

The authors have no conflicts to disclose.

\section{REFERENCES}

1. Evans AA, London WT, Gish RG, Cohen C, Block TM. Chronic HBV infection outside treatment guidelines: is treatment needed? Antivir Ther 2013;18:229-235.

2. Chen CJ, Yang HI, Su J, Jen CL, You SL, Lu SN, et al. Risk of hepatocellular carcinoma across a biological gradient of serum hepatitis $B$ virus DNA level. JAMA 2006;295:65-73.

3. Iloeje UH, Yang HI, Su J, Jen CL, You SL, Chen CJ. Predicting cirrhosis risk based on the level of circulating hepatitis B viral load. Gastroenterology 2006;130:678-686.

4. Hosaka T, Suzuki F, Kobayashi M, Seko Y, Kawamura Y, Sezaki H, et al. Long-term entecavir treatment reduces hepatocellular carcinoma incidence in patients with hepatitis B virus infection. Hepatology 2013;58:98-107.

5. Lai CL, Yuen MF. Prevention of hepatitis B virus-related hepatocellular carcinoma with antiviral therapy. Hepatology 2013;57:399-408.

6. Chang TT, Liaw YF, Wu SS, Schiff E, Han KH, Lai CL, et al. Long-term entecavir therapy results in the reversal of fibrosis/cirrhosis and continued histological improvement in patients with chronic hepatitis B. Hepatology 2010;52:886-893.

7. Marcellin P, Gane E, Buti M, Afdhal N, Sievert W, Jacobson IM, et al. Regression of cirrhosis during treatment with tenofovir disoproxil fumarate for chronic hepatitis B: a 5-year open-label follow-up study. Lancet 2013;381:468-475.

8. Liaw YF, Sung JJ, Chow WC, Farrell G, Lee CZ, Yuen H, et al. Lamivudine for patients with chronic hepatitis $B$ and advanced liver disease. N Engl J Med 2004;351:1521-1531.

9. Lok AS, Lai CL, Leung N, Yao GB, Cui ZY, Schiff ER, et al. Long-term safety of lamivudine treatment in patients with chronic hepatitis $B$. Gastroenterology 2003;125:1714-1722.

10. Yuen MF, Sablon E, Hui CK, Yuan HJ, Decraemer H, Lai CL. Factors associated with hepatitis $B$ virus DNA breakthrough in patients receiving prolonged lamivudine therapy. Hepatology 2001;34:785791.

11. Lee YS, Suh DJ, Lim YS, Jung SW, Kim KM, Lee HC, et al. Increased risk of adefovir resistance in patients with lamivudine-resistant chronic hepatitis B after 48 weeks of adefovir dipivoxil monotherapy. Hepatology 2006;43:1385-1391.

12. Yim HJ, Hussain M, Liu Y, Wong SN, Fung SK, Lok AS. Evolution of multi-drug resistant hepatitis $B$ virus during sequential therapy. Hepatology 2006;44:703-712.

13. Liu Y, Wang C, Zhong Y, Chen L, Li X, Ji D, et al. Evolution and suppression of HBV strains with multidrug resistance to lamivudine, adefovir dipivoxil and entecavir in a patient with chronic hepatitis B. Antivir Ther 2010;15:1185-1190.

14. Lok AS, McMahon BJ. Chronic hepatitis B: update 2009. Hepatology 2009;50:661-662.

15. EASL clinical practice guidelines: Management of chronic hepatitis $B$ virus infection. J Hepatol 2012;57:167-185.

16. Kitrinos KM, Corsa A, Liu Y, Flaherty J, Snow-Lampart A, Marcellin P, et al. No detectable resistance to tenofovir disoproxil fumarate after 6 years of therapy in patients with chronic hepatitis B. Hepatology 2014;59:434-442.

17. Delaney WE 4th, Ray AS, Yang H, Qi X, Xiong S, Zhu Y, et al. Intracellular metabolism and in vitro activity of tenofovir against hepatitis B virus. Antimicrob Agents Chemother 2006;50:2471-2477.

18. Hann HW, Chae HB, Dunn SR. Tenofovir (TDF) has stronger antiviral effect than adefovir (ADV) against lamivudine (LAM)-resistant hepatitis B virus (HBV). Hepatol Int 2008;2:244-249.

19. Kuo A, Dienstag JL, Chung RT. Tenofovir disoproxil fumarate for the treatment of lamivudine-resistant hepatitis B. Clin Gastroenterol Hepatol 2004;2:266-272.

20. van Bömmel F, de Man RA, Wedemeyer H, Deterding K, Petersen J, Buggisch $P$, et al. Long-term efficacy of tenofovir monotherapy for hepatitis $B$ virus-monoinfected patients after failure of nucleoside/ nucleotide analogues. Hepatology 2010;51:73-80.

21. van Bömmel $F$, Wünsche T, Mauss $S$, Reinke $P$, Bergk $A$, Schürmann $D$, et al. Comparison of adefovir and tenofovir in the treatment of lamivudine-resistant hepatitis B virus infection. Hepatology 2004;40:1421-1425.

22. Berg T, Marcellin P, Zoulim F, Moller B, Trinh $H$, Chan S, et al. Tenofovir is effective alone or with emtricitabine in adefovir-treated patients with chronic-hepatitis B virus infection. Gastroenterology 2010;139:1207-1217.

23. Berg T, Zoulim F, Moeller B, Trinh H, Marcellin P, Chan S, et al. Longterm efficacy and safety of emtricitabine plus tenofovir DF vs. tenofovir DF monotherapy in adefovir-experienced chronic hepatitis B patients. J Hepatol 2014;60:715-722.

24. Patterson SJ, George J, Strasser SI, Lee AU, Sievert W, Nicoll AJ, et al. Tenofovir disoproxil fumarate rescue therapy following failure of both lamivudine and adefovir dipivoxil in chronic hepatitis B. Gut 2011;60:247-254

25. Fung S, Kwan P, Fabri M, Horban A, Pelemis M, Hann HW, et al. Randomized comparison of tenofovir disoproxil fumarate vs emtricitabine and tenofovir disoproxil fumarate in patients with lamivudine-resistant chronic hepatitis B. Gastroenterology 2014;146:980988.

26. Zoutendijk R, Reijnders JG, Brown A, Zoulim F, Mutimer D, Deterding $\mathrm{K}$, et al. Entecavir treatment for chronic hepatitis B: adaptation is not needed for the majority of naive patients with a partial virological response. Hepatology 2011;54:443-451. 
27. Yuen MF, Seto WK, Fung J, Wong DK, Yuen JC, Lai CL. Three years of continuous entecavir therapy in treatment-naive chronic hepatitis B patients: VIRAL suppression, viral resistance, and clinical safety. Am J Gastroenterol 2011;106:1264-1271.

28. Ono A, Suzuki F, Kawamura Y, Sezaki H, Hosaka T, Akuta N, et al. Long-term continuous entecavir therapy in nucleos(t)ide-naive chronic hepatitis B patients. J Hepatol 2012;57:508-514.

29. Shin JW, Jung SW, Park BR, Kim CJ, Eum JB, Kim BG, et al. Prediction of response to entecavir therapy in patients with $\mathrm{HBeAg}$ positive chronic hepatitis B based on on-treatment $\mathrm{HBsAg}, \mathrm{HBeAg}$ and HBV DNA levels. J Viral Hepat 2012;19:724-731.

30. Gordon SC, Krastev Z, Horban A, Petersen J, Sperl J, Dinh P, et al. Efficacy of tenofovir disoproxil fumarate at 240 weeks in patients with chronic hepatitis B with high baseline viral load. Hepatology 2013;58:505-513.
31. Pol S, Lampertico P. First-line treatment of chronic hepatitis B with entecavir or tenofovir in 'real-life' settings: from clinical trials to clinical practice. J Viral Hepat 2012;19:377-386.

32. Hongthanakorn C, Chotiyaputta W, Oberhelman K, Fontana RJ, Marrero JA, Licari T, et al. Virological breakthrough and resistance in patients with chronic hepatitis $B$ receiving nucleos(t)ide analogues in clinical practice. Hepatology 2011;53:1854-1863.

33. Lok AS, Trinh H, Carosi G, Akarca US, Gadano A, Habersetzer F, et al. Efficacy of entecavir with or without tenofovir disoproxil fumarate for nucleos(t)ide-naive patients with chronic hepatitis B. Gastroenterology 2012;143:619-628, e1.

34. Chotiyaputta W, Hongthanakorn C, Oberhelman K, Fontana RJ, Licari T, Lok AS. Adherence to nucleos(t)ide analogues for chronic hepatitis B in clinical practice and correlation with virological breakthroughs. J Viral Hepat 2012;19:205-212. 